\title{
Neutral-Plasma Oscillations at Zero Temperature
}

\author{
Scott D. Bergeson \\ scott.bergeson@byu.edu \\ Ross L. Spencer \\ ross_spencer@byu.edu
}

Follow this and additional works at: https://scholarsarchive.byu.edu/facpub

Part of the Astrophysics and Astronomy Commons, and the Physics Commons

\section{Original Publication Citation}

S. D. Bergeson and R. L. Spencer, "Neutral plasma oscillations at zero temperature," Physical Review E 67, 26414, pp. 1-5, (23). The original publication of this article can be found at http://link.aps.org/doi/1.113/PhysRevE.67.26414

\section{BYU ScholarsArchive Citation}

Bergeson, Scott D. and Spencer, Ross L., "Neutral-Plasma Oscillations at Zero Temperature" (2003).

Faculty Publications. 513.

https://scholarsarchive.byu.edu/facpub/513

This Peer-Reviewed Article is brought to you for free and open access by BYU ScholarsArchive. It has been accepted for inclusion in Faculty Publications by an authorized administrator of BYU ScholarsArchive. For more information, please contact ellen_amatangelo@byu.edu. 


\title{
Neutral-plasma oscillations at zero temperature
}

\author{
S. D. Bergeson and R. L. Spencer \\ Department of Physics and Astronomy, Brigham Young University, Provo, Utah 84602 \\ (Received 29 April 2002; revised manuscript received 16 October 2002; published 26 February 2003)

\begin{abstract}
Cold plasma theory is used to calculate the response of an ultracold neutral plasma to an applied rf field. The free oscillation of the system has a continuous spectrum and an associated damped quasimode. This quasimode dominates the driven response and is resonant in the tail of the density distribution. Recent experiments used the plasma response to an applied rf field to determine the plasma density in an expanding ultracold plasma. The comparison between experiment and theory indicates that this method accurately determines the expansion velocity and underestimates the initial plasma density by a factor of 3 in weakly collisional plasmas.
\end{abstract}

DOI: 10.1103/PhysRevE.67.026414

PACS number(s): 52.27.Gr, 52.55.Dy, 52.35.Fp

\section{INTRODUCTION}

Ultracold plasmas are a relatively new extension to the field of laser cooling [1]. These plasmas are created either by directly photoionizing laser-cooled atoms [2-4], or by exciting laser-cooled atoms to high-lying Rydberg states that are subsequently ionized $[5,6]$. By choosing the initial electron and ion (or Rydberg atom) temperatures and densities, it is possible to precisely control the initial conditions of a very strongly interacting system.

A recently published paper describes the expansion of an ultracold neutral plasma [3]. Xenon atoms were initially cooled to $\sim 10 \mu \mathrm{K}$ and then ionized by a dye laser pulse. A small charge imbalance in the plasma produced an electric field that drove a slow ion expansion. The expanding plasma was probed with a spatially uniform radio-frequency ( $\mathrm{rf}$ ) electric field. By making some simple assumptions about how the plasma reacted to the field, it was possible to determine an average plasma density, and to see how that density changed with time.

In this paper we analyze in more detail how an ultracold plasma responds to an rf field. We first derive the mode response of the system predicted by cold plasma theory. We then choose an expansion model and ask whether or not the known expansion can be determined from the plasma's response to the rf field.

Surprisingly, this system has no normal modes. Instead, it has a continuous spectrum with an associated damped quasimode that is resonant in the tail of the density distribution, shifting the response to lower densities than expected. This means that the average densities determined in this way underestimate the actual density.

\section{THE PLASMA RESPONSE TO A RF FIELD}

The greatest response of a plasma to an rf field occurs when the rf frequency is on the order of the plasma frequency. Because the plasma frequency is proportional to the square root of density, determining the plasma response to an applied rf field requires first knowing the plasma density.

A number of ultracold plasma models have been published [7-13]. The work of Robicheaux and Hanson [11] deals particularly with the density profile of the expanding plasma. They write the density for the neutral plasma as $n(r, t)=N_{i}[\beta(t) / \pi]^{3 / 2} \exp \left[-\beta(t) r^{2}\right]$, where $N_{i}$ is the number of ions. The function $\beta(t)$ is given by Eq. (2) in their paper, and can be solved analytically. Using their boundary conditions, we find $\beta(t)=1 / 2\left(\sigma_{0}^{2}+v^{2} t^{2}\right)$, making the density profile

$$
n(r, t)=\frac{n_{0} \sigma_{0}^{3}}{\sigma^{3}} \exp \left[-r^{2} / 2 \sigma^{2}\right]
$$

where $n_{0}$ is the initial density at the center of the plasma, $\sigma_{0}$ is the initial width of the distribution, $\sigma=\sqrt{\sigma_{0}^{2}+v^{2} t^{2}}$ is the time-dependent width of the distribution, $v=\sqrt{E_{e} / k_{B}}$ is the asymptotic expansion velocity, $E_{e}$ is the initial electron energy, $k_{B}$ is Boltzmann's constant, and $t$ is time. Note that this is the formula for a self-similar Gaussian expansion and is, in fact, the profile assumed in the initial experimental work of Ref. [3].

We therefore consider a spherically symmetric chargeneutral Gaussian distribution of cold ions and electrons to which an rf-electric field in the $z$ direction is applied. The ions are taken as fixed on the time scale of the rf oscillation. The cold fluid equations describing the electrons are

$$
\begin{gathered}
\frac{\partial n}{\partial t}+\vec{\nabla} \cdot(n \vec{v})=0, \\
\nabla^{2} \phi=-q\left(n-n_{i}\right) / \epsilon_{0}, \\
m\left(\frac{\partial \vec{v}}{\partial t}+\vec{v} \cdot \vec{\nabla} \vec{v}\right)=q \vec{E}-\nu m \vec{v}
\end{gathered}
$$

In these equations $n=n(r, t)$ is the electron density, $n_{i}$ $=n_{i}(r)$ is the ion density, $\vec{v}=\vec{v}(\vec{r}, t)$ is the electron velocity, $\vec{E}=\vec{E}(\vec{r}, t)$ is the total electric field (the sum of the external field and that generated by the plasma response), and $\nu$ is a damping rate that includes some effects of finite temperature, such as electron-electron collisions and electron-ion collisions. This theory is good for plasmas in which $\left(\lambda_{D} / \sigma\right)^{2}$ $\ll 1$, where $\lambda_{D}$ is the Debye length. In the experiments we 
are simulating, $\left(\lambda_{D} / \sigma\right)^{2} \approx 10^{-3}-10^{-1}$, making cold plasma theory a good way to model the collective response of the system.

In the experiment by Kulin et al. [3], the applied $\vec{E}$ was uniform in space and oscillating in the $\hat{z}$ direction. If the applied field is small, the fluid equations can be linearized by assuming that the density and the potential are of the form $n(r, \theta, t)=n^{(0)}(r)+\delta n(r) \cos \theta e^{-i \omega t}$, where $n^{(0)}(r)$ is the equilibrium electron density and $\phi(r, \theta, t)$ $=\delta \phi(r) \cos \theta e^{-i \omega t}$ (spherical harmonics with $\ell=1$ ). After some algebra to linearize the fluid equations (2)-(4), the potential produced by the electrons is given by the expression

$$
\mathcal{L} \delta \phi-\frac{\left[\frac{d}{d r} \omega_{p}^{2}(r)\right] \frac{\partial \delta \phi}{\partial r}}{\omega^{2}-\omega_{p}^{2}(r)+i \omega \nu}=-\frac{\left[\frac{d}{d r} \omega_{p}^{2}(r)\right] E_{0}}{\omega^{2}-\omega_{p}^{2}(r)+i \omega \nu},
$$

where the operator $\mathcal{L}$ is defined as

$$
\mathcal{L} \equiv \frac{d^{2}}{d r^{2}}+\frac{2}{r} \frac{d}{d r}-\frac{2}{r^{2}}
$$

and where $\omega_{p}(r)=\sqrt{q^{2} n^{(0)}(r) / m_{e} \epsilon_{0}}$ is the plasma frequency, $q$ is the electron charge, $m_{e}$ is the electron mass, and $\epsilon_{0}$ is the permittivity of free space. Note that because the density varies with radius, the local plasma frequency varies as well. The boundary conditions on the potential are that $\delta \phi(0)=0$ and that at infinity the field is that of a dipole, $\delta \phi(r) \propto 1 / r^{2}$.

Because this is a driven system, we might first look for normal modes with which to resonate. Notice, however, that with $E_{0}=0$ and $\nu=0$ (undamped free response) this mode equation has a continuous spectrum instead of discrete modes. This is similar to the diocotron mode equation in non-neutral plasmas [14-19]. And, as with the diocotron mode, this equation also has a damped quasimode. Following Ref. [15], Eq. (5) can be solved along a contour in the complex $r$ plane to uncover this damped quasimode. Figure 1 shows the frequency and damping rate of this quasimode for a sequence of density profiles of the form

$$
n(r)=n_{0} e^{-r^{p} / 2 \sigma^{p}} .
$$

The real and imaginary parts of the quasimode frequency are well approximated by the simple formulas $\omega_{m} / \omega_{p}(0)$ $=1 / \sqrt{3}-0.668 / p-0.102 / p^{2} \quad$ and $\quad \nu_{m} / \omega_{p}(0)=1.077 / p$ $-0.959 / p^{2}$.

Note that the Gaussian $(p=2)$ quasimode is heavily damped and that as $p$ becomes large (making the density profile approach a step profile), the damping rate goes to zero and the frequency approaches $\omega_{m}=\omega_{p}(0) / \sqrt{3}$. The factor $1 / \sqrt{3}$ in the high- $p$ limit is the well-known correction to the plasma frequency for a finite plasma in spherical geometry.

The quasimode dominates the free response of a system with a continuous spectrum [20,21]. It also strongly influences the driven response, as can be seen in Fig. 2. This figure shows the rate at which a plasma absorbs power from an applied rf field as a function of the frequency of that field.

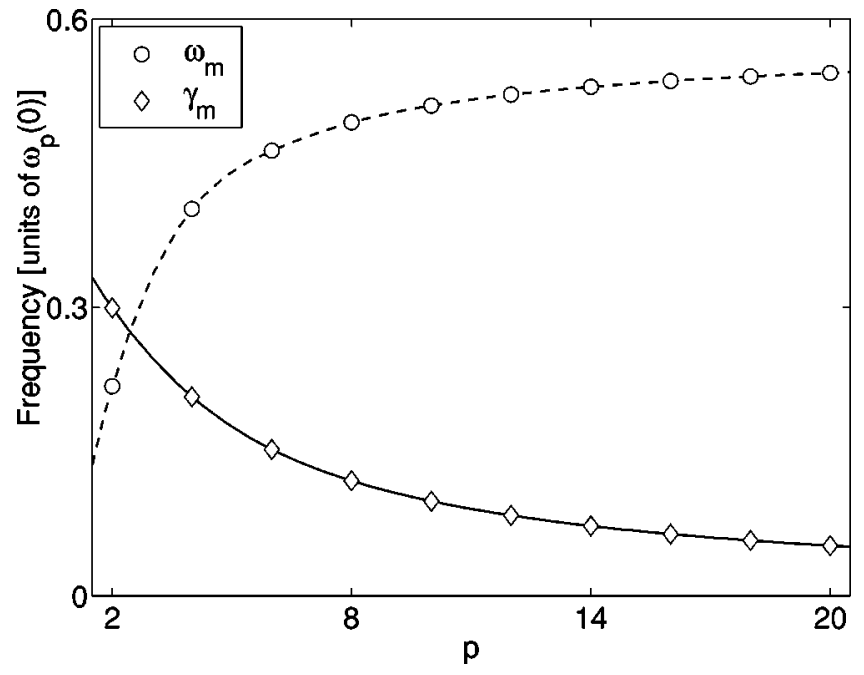

FIG. 1. Quasimode frequency $\omega_{m}(\bigcirc)$ and damping rate $\nu_{m}$ $(\diamond)$, for density profiles given by Eq. (7). For $p=2$, the distribution is Gaussian. As $p$ increases, the distribution approaches a "top hat," the quasimode frequency increases, and the quasimode damping decreases (see text).

To calculate this power, we note that the total electric field (the applied field plus the plasma response) is given by

$$
\vec{E}(r, t)=\left(\hat{z} E_{0} \cos \theta-\vec{\nabla} \delta \phi\right) e^{-i \omega t} .
$$

By using the linearized Eq. (4) to compute the current density $\vec{J}=q n \vec{v}$ in the plasma, the power density can be written as

$$
\langle P\rangle=\frac{1}{2} \operatorname{Re}\left(\vec{E} \cdot \vec{J}^{*}\right)=\frac{\epsilon_{0}}{2}|\vec{E}|^{2} \frac{\omega_{p}^{2}(r) \nu}{\omega^{2}+\nu^{2}}
$$

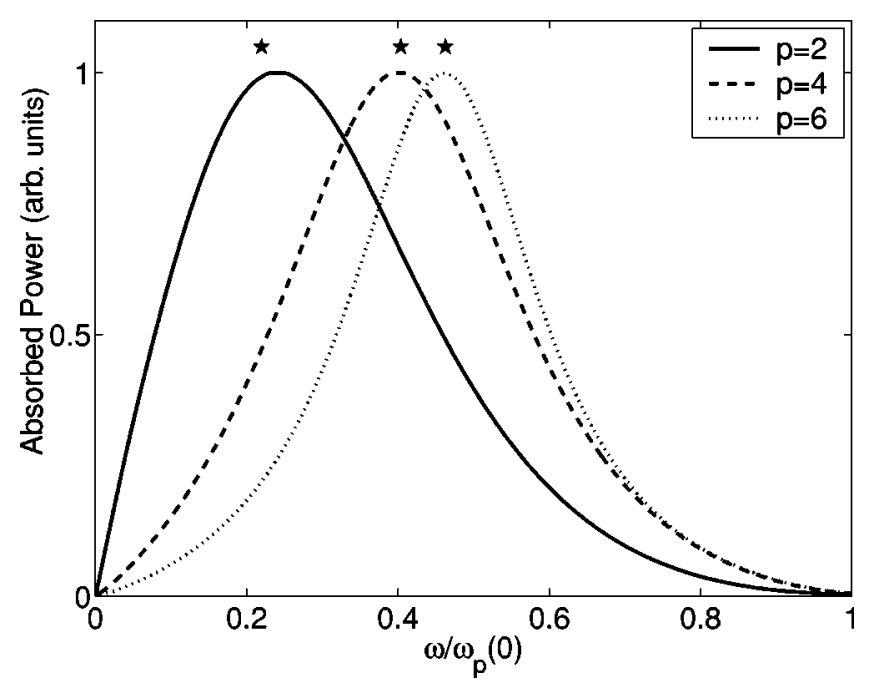

FIG. 2. Power absorbed by the (nonexpanding) plasma as a function of applied frequency. The solid line shows the absorbed power for a Gaussian density profile, $p=2$ in Eq. (7). Also shown is the absorbed power for super-Gaussian profiles, with $p=4,6$. The mode damping decreases with increasing $p$. These plots are generated with $\nu / \omega_{p}^{(0)}=0.01$. For larger values of $\nu$, these curves are somewhat broader and shifted to slightly higher $\omega$. 
The influence of the quasimode on the absorbed power can be seen by integrating this power density over all space for various values of the driving frequency $\omega$. Although strongly damped, the quasimode still influences how the plasma responds to the rf field. Figure 2 shows the calculated power absorbed by the plasma as a function of the applied rf frequency for a nonexpanding plasma with weak damping. In each case the peak of the absorbed power occurs near the frequency of the quasimode. Notice that these resonances occur at quite small values of the density, rather than in the body of the density distribution as assumed by Kulin et al. The experiment used $\omega / \omega_{p}(0)=0.6$.

In the following section, we will interpret the experiments of Ref. [3] in terms of this theory. Among other things, it will be seen that the quasimode resonance shifts the plasma response to lower densities than might otherwise be expected.

\section{THE RELATIONSHIP BETWEEN TEMPERATURE AND DENSITY}

To apply this theory to experiments, we need to know how $\nu$ varies in the expansion. This requires an understanding of how density and temperature are related.

Without going into the details of heating (three-body recombination, electron-Rydberg scattering, etc.) or cooling (electron evaporation, electron-ion collisions, expansion, etc.), we will assume that the electron temperature scales with density as $T \propto n^{\alpha}$. This choice is motivated in part by the recent work of Ref. [11]. Note that the coefficient $\alpha$ could have time or density dependence to include heating and/or cooling effects. In this work, we will take it to be a constant. However we find that for the weakly collisional plasmas we are simulating, there is essentially no dependence on $\alpha$ over the most physically plausible range.

The damping rate $\nu$ is probably dominated by the electron collision frequency, $\nu_{e i}$. Defining the electron collision impact parameter to be $b=q^{2} / 4 \pi \epsilon_{0} k_{B} T$, the electron collision frequency can be written as

$$
\nu_{e i}=n \pi b^{2} \lambda_{D} \omega_{p} \ln \left(\frac{\lambda_{D}}{b}\right)
$$

where $\ln \left(\lambda_{D} / b\right)$ is the Coulomb logarithm.

By assuming $T \propto n^{\alpha}$, the density dependence of the collision term can be made more explicit:

$$
\nu_{e i}=\nu_{e i}^{0}\left(\frac{n}{n^{0}}\right)^{1-3 \alpha / 2}\left[1+\left(\frac{3 \alpha-1}{2}\right) \frac{\ln \left(n / n^{0}\right)}{\ln \left(\lambda_{D}^{0} / b^{0}\right)}\right] .
$$

In this equation, $\nu_{e i}^{0}, \lambda_{D}^{0}$, and $b^{0}$ are the values of the collision frequency, the Debye length, and impact parameter in the center of the plasma at the beginning of the expansion.

For an ideal gas undergoing adiabatic expansion, $\alpha$ $=2 / 3$. However the expanding ultracold plasma is probably not expanding adiabatically. The three-body recombination rate in weakly coupled plasmas scales as $\kappa_{3} \sim n^{2} T^{-9 / 2}$ $\sim n^{(4-9 \alpha) / 2}$. If the plasma expanded as an ideal gas, the three-body recombination rate would be $\kappa_{3} \sim n^{-1}$. So even if the plasma is created in a density and temperature regime where recombination is ignorable, it quickly cools to the point where recombination turns on, making the expansion nonadiabatic [11]. Note that any value of $\alpha<4 / 9$ avoids the problem of recombination becoming too large an effect during the expansion.

A more realistic relationship between density and temperature from Ref. [11] uses $\alpha=1 / 3$. This has the consequence that the strong coupling parameter in the expanding plasma remains roughly constant $[11,13]$. It also means that the Coulomb logarithm is independent of density, and that $\nu / \omega_{p}$ is constant during the expansion. The model used to determine this value of $\alpha$ considers three-body recombination and electron-Rydberg scattering as the main heating sources. There may also be other heating sources that give smaller values for $\alpha$.

\section{THE RESPONSE OF AN EXPANDING PLASMA}

With a choice for $\alpha$, it is now possible to use the above analysis to simulate the response of an expanding plasma to an rf field. We mimic the experiment [3] by fixing the frequency of the rf field and letting the plasma expand. For the purpose of comparing to experiment, we define the average plasma density to be

$$
\begin{aligned}
\bar{n}(t) & =\frac{\int[n(r, t)]^{2} 4 \pi r^{2} d r}{\int n(r, t) 4 \pi r^{2} d r} \\
& =\frac{n_{0} \sigma_{0}^{3}}{\left[2\left(\sigma_{0}^{2}+v^{2} t^{2}\right)\right]^{3 / 2}} \\
& =2^{-3 / 2} n(0, t),
\end{aligned}
$$

with $n(r, t)$ defined by Eq. (1). We also define the average plasma frequency to be

$$
\bar{\omega}_{p}(t)=\sqrt{\frac{\left.q^{2} \overline{n(} t\right)}{m_{e} \epsilon_{0}}} .
$$

In the experiment, they assumed that the maximum response of the plasma occurred when the driving field resonated with the average plasma frequency $\underline{\omega_{p}}$, and used that response to determine the average density $n$.

We simulate the plasma expansion experiment as follows. The plasma expansion velocity $v$, the rf frequency $\omega$, and $\mathrm{rf}$ amplitude $E_{0}$ are held constant. At a particular time $t$, we insert the density from Eq. (1) into Eq. (5) to find the potential $\delta \phi$ and the total electric field [Eq. (8)]. We integrate the power density [Eq. (9)] over the spatial coordinates to get the plasma heating rate at that time. We increment time and repeat the heating rate calculation to generate one of the time sweeps shown in Fig. 3(a). Repeating these calculations for a 

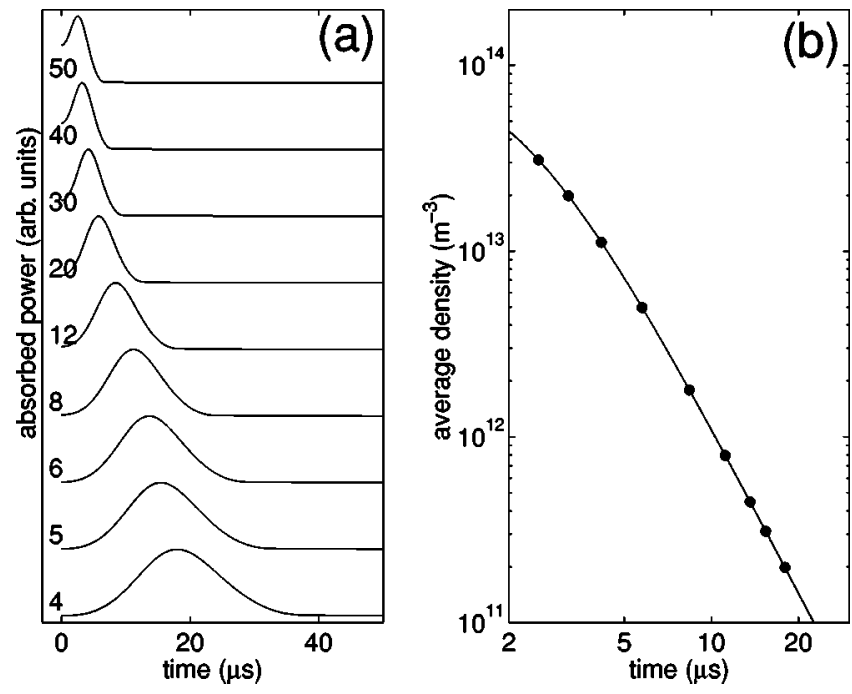

FIG. 3. (a) Calculated plasma heating rate. The applied frequencies $\omega / 2 \pi$ in megahertz are shown to the left of each trace. The traces are offset vertically for clarity. The plasma conditions are $n_{0}=10^{15} \mathrm{~m}^{-3}, v=100 \mathrm{~m} / \mathrm{s}, \sigma_{0}=220 \mu \mathrm{m}$, and $\nu / \omega_{p}(0)=0.01$. (b) Average plasma density as a function of time. The circles $(\bullet)$ are taken from the data in (a). The applied frequency is converted to a density $\bar{n}(t)$, with $t$ given by the peak of the signal in (a) (see text). The solid line is a fit of these points to Eq. (12), with $\bar{n}(0)$ and $v$ as free parameters. The fitted density is $\bar{n}(0)=1.1 \times 10^{14} \mathrm{~m}^{-3}$ or $n_{0}$ $=3.1 \times 10^{14} \mathrm{~m}^{-3}$, about a factor of 3 too low. The fitted expansion velocity is $v=100 \mathrm{~m} / \mathrm{s}$, in exact agreement with the expansion model.

range of applied field frequencies generates all of the time sweeps shown in the figure.

We convert the time sweeps in Fig. 3(a) into average density determinations. At the peak of each time sweep we set the applied frequency $\omega$ equal to the average plasma frequency [Eq. (13)], to calculate the average density $\bar{n}$, as was done in the experiment [3]. With this conversion from frequency to density, the simulated average density $\bar{n}(t)$ is plotted in Fig. 3(b). The data is fit to Eq. (12) using a leastsquares method to extract the initial central density $n_{0}$ and expansion velocity $v$.

\section{DISCUSSION}

The objective of this paper is to find out if the density determined in this fashion accurately reproduces the known density from the expansion model. With the initial value of the damping rate set by the electron-electron collision rate, the only free parameter in the model is $\alpha$, the exponent in the relationship between density and temperature $\left(T \propto n^{\alpha}\right)$.

For the conditions given in the original experiment $\left(\nu^{(0)} / \omega_{p}^{(0)}=0.01\right)$, we find that the derived density and expansion velocity are independent of $\alpha$ for physically reasonable values in the range 4/9> $\alpha>0$. The shapes of the calculated plasma heating rates shown in Fig. 3(a) match the experimental ones from Ref. [3], and the fit of $\bar{n}(t)$ in Fig. 3 (b) to Eq. (12) is excellent.

The fitted density extracted from Fig. 3 is about a factor of 3 lower than the density used in the expansion model. It is a little surprising that the fitted density is not lower. After all, the quasimode resonance occurs at a frequency three times lower than expected, which should translate into a factor of 9 in the density. Apparently, averaging the quasimode resonance over the density distribution shifts the peak plasma heating rate to somewhat higher densities.

Cold plasma theory only addresses the collective behavior of the system. Another effect that might be involved in the resonant behavior seen in the experiment is that the electron motion in the plasma resonates with the driving field. Robicheaux and Hanson estimate that in the bulk of the plasma, the potential satisfies $q \phi / k_{B} T=r^{2} / 2 \sigma^{2}$. This means that most of the particles are in an isotropic harmonic oscillator well with natural frequency $\omega_{0}=\omega_{p}(0) \lambda_{D}(0) / \sigma$, where $\lambda_{D}(0)$ is the Debye length at the center of the cloud. Since $\lambda_{D}(0) \sim 1 / 10$ and $\omega \sim \omega_{p}(0) / 5$, perhaps kinetic effects are important. But such effects are beyond the scope of this theoretical treatment.

Cold plasma theory is valid for determining the collective response of quasineutral plasmas in which $(\lambda / \sigma)^{2} \ll 1$. This is true for the cold plasma experiments of Ref. [3]. At lower temperatures, electron-ion recombination, electron-Rydberg scattering, and strong plasma coupling significantly alter the plasma behavior, as discussed by other authors [11-13]. Our treatment also explicitly assumes that the driving field is weak compared to the field internal to the plasma resulting from a small charge imbalance, such as is true for the experiments we are simulating.

\section{CONCLUSION}

We have used a simulation based on cold plasma theory to calculate the response of an expanding ultracold plasma to an applied rf field. The plasma response to the field is dominated by the quasimode. Even though the mode is strongly damped, it shifts the plasma resonance to lower densities than otherwise expected from a local-density approximation. In using the theory to interpret experimental expansion data, we assumed the relationship between temperature and density to be $T \propto n^{\alpha}$, although the expansion was independent of the particular value of $\alpha$ across its most likely range. The plasmas we simulate are initially weakly collisional, and remain so as they expand. The expansion velocity determined by the response of the plasma to the field accurately reproduces the actual plasma expansion velocity. The initial density determined from the plasma response to the field gives the proper order of magnitude, and underestimates the actual density by a factor of 3 .

\section{ACKNOWLEDGMENTS}

This work was supported in part by grants from the Research Corporation and from the National Science Foundation (Grant No. PHY-9985027). 
[1] S.L. Rolston, S.D. Bergeson, S. Kulin, and C. Orzel, Bull. Am. Phys. Soc. 43, 1324 (1998).

[2] T.C. Killian, S. Kulin, S.D. Bergeson, L.A. Orozco, C. Orzel, and S.L. Rolston, Phys. Rev. Lett. 83, 4776 (1999).

[3] S. Kulin, T.C. Killian, S.D. Bergeson, and S.L. Rolston, Phys. Rev. Lett. 85, 318 (2000).

[4] T.C. Killian, M.J. Lim, S. Kulin, R. Dumke, S.D. Bergeson, and S.L. Rolston, Phys. Rev. Lett. 86, 3759 (2001).

[5] M.P. Robinson, B. Laburthe Tolra, Michael W. Noel, T.F. Gallagher, and P. Pillet, Phys. Rev. Lett. 85, 4466 (2000).

[6] S.K. Dutta, D. Feldbaum, A. Walz-Flannigan, J.R. Guest, and G. Raithel, Phys. Rev. Lett. 86, 3993 (2001).

[7] R. Côté and A. Dalgarno, Phys. Rev. A 62, 012709 (2000).

[8] C.H. Greene, A.S. Dickinson, and H.R. Sadeghpour, Phys. Rev. Lett. 85, 2458 (2000).

[9] A.N. Tkachev and S.I. Yakovlenko, JETP Lett. 73, 66 (2001).

[10] Y. Hahn, Phys. Rev. E 64, 046409 (2001).

[11] F. Robicheaux and J.D. Hanson, Phys. Rev. Lett. 88, 055002 (2002).

[12] S. Mazevet, L.A. Collins, and J.D. Kress, Phys. Rev. Lett. 88,
055001 (2002).

[13] S.G. Kuzmin and T.M. O’Neil, Phys. Rev. Lett. 88, 065003 (2002).

[14] D. A. Schecter, D. H. E. Dubin, A. Cass, C. F. Driscoll, I. M. Lansky, and T. M. O'Neil, in Non-Neutral Plasma Physics III, edited by John J. Bollinger et al. (AIP, New York, 1999).

[15] R.L. Spencer and S.N. Rasband, Phys. Plasmas 4, 53 (1997).

[16] R.J. Briggs, J.D. Daugherty, and R.H. Levy, Phys. Fluids 13, 421 (1970).

[17] K.M. Case, Phys. Fluids 3, 143 (1960).

[18] W. Kelvin, Nature (London) 23, 45 (1880).

[19] N.R. Corngold, Phys. Plasmas 2, 620 (1995).

[20] D.A. Schecter, D.H.E. Dubin, A.C. Cass, C.F. Driscoll, I.M. Lansky, and T.M. O’Neil, Phys. Fluids 12, 2397 (2000).

[21] D.A. Schecter, D.H.E. Dubin, A.C. Cass, C.F. Driscoll, I.M. Lansky, and T.M. O'Neil, in Non-Neutral Plasma Physics III, edited by John J. Bollinger, Ross L. Spencer, and Ronald C. Davidson, AIP Conf. Proc. No. 498 (AIP, Meville, NY, 1999), p. 115 . 